\title{
REABILITAÇÃO DE ACIDENTE VASCULAR ENCEFÁLICO: REVISÃO DE LITERATURA
}

\author{
REHABILITATION OF STROKE: LITERATURE REVIEW
}

\section{Daiana Cristina Dessuy Vieira ${ }^{a}$, Carine Cristina Callegaro ${ }^{b}$, Vivian Lemes Lobo Bittencourtc, Taís Paz da Silva ${ }^{d}$, Eliane Roseli Winkelmanne}

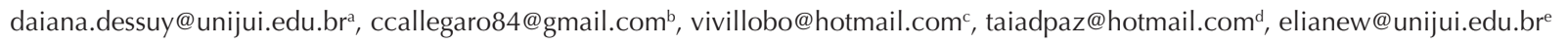
Universidade Regional do Noroeste do Rio Grande do Sul - ljuí (RS), Brasil.

Data de recebimento do artigo: 09/02/2017 Data de aceite do artigo: 03/05/2017

\section{RESUMO}

Introdução: A prevalência do acidente vascular encefálico é alta, e $90 \%$ dos sobreviventes desenvolvem algum tipo de deficiência ou incapacidade, com prejuízos nas funçôes sensitivas, motoras, de equilíbrio e de marcha. As avaliaçóes clínicas e instrumentais especializadas devem ser utilizadas na prática interdisciplinar, a fim de diagnosticar o nível de comprometimento, as potencialidades, e definir o plano de cuidados, fundamentado em métodos destinados à recuperaçáo de déficits ou desenvolvimento de compensaçôes. Objetivo: Identificar na literatura evidências da eficácia das intervençôes utilizadas por equipe interdisciplinar na reabilitação do paciente pós-acidente vascular encefálico, bem como fatores que influenciam no acesso e entrega da reabilitação. Materiais e Métodos: Revisão integrativa da literatura, utilizando artigos publicados entre 2010 e 2015 nas bases de dados Pubmed, Lilacs, Scielo e Cochrane Library. Utilizou-se como descritores: acidente vascular encefálico, reabilitação e equipe de assistência ao paciente. Resultados: Foram incluídos 12 estudos, e observou-se fatores decisivos para acesso e exclusão do indivíduo na reabilitação e a carência de estudos que comprovem a eficácia da reabilitação. Porém, a reabilitação melhora os resultados funcionais, desde que seja bem estruturada e atuante na prevenção e educação da família. Conclusóes: A reabilitação é um processo continuado e integral. É importante a participação conjunta da equipe interdisciplinar, da família e da comunidade na continuidade dos cuidados.

Palavras-chave: Acidente vascular encefálico; reabilitação; equipe de assistência ao paciente.

\section{ABSTRACT}

Introduction: The prevalence of stroke is high and $90 \%$ of survivors develop some type of disability or disability, with impairment of sensory, motor, balance and gait function. Specialized clinical and instrumental evaluations should be used in interdisciplinary practice in order to diagnose the level of commitment, the potentialities, and define the care plan, based on methods for the recovery of deficits or the development of compensations. Objective: To identify in the literature evidences of the effectiveness of the interventions used by interdisciplinary team in the rehabilitation of the patient after stroke, as well as factors that influence the access and delivery of the rehabilitation. Materials and Methods: Integrative review of the literature, from articles published between 2010 and 2015 in the databases: PubMed, Lilacs, Scielo and Cochrane Library. The following were used as descriptors: stroke, rehabilitation and patient care team. Results: 12 studies were included, decisive factors for access and exclusion of the individual in rehabilitation, lack of studies proving the effectiveness of rehabilitation, but rehabilitation improves functional results provided that it is well structured and active in prevention and education of the family. Conclusions: Rehabilitation is a continuous and integral process. It is important the joint participation of the interdisciplinary team, the family and the community in the continuity of care.

Keywords: Stroke; rehabilitation; patient care team. 


\section{Introdução}

No decorrer das últimas décadas, a humanidade tornou-se mais populosa, principalmente com o crescente número de indivíduos com mais de 60 anos de idade. No Brasil, essa faixa da população cresceu $33,65 \%$ no período de 2000 a 2009 , e com esse crescimento, incidem e predominam as doenças crônicas não transmissíveis ${ }^{1}$.

Destaca-se nesta população a alta prevalência de doenças cardiovasculares (DCV), como a doença cardíaca coronariana e o acidente vascular encefálico (AVE), que permanecem como as principais causas de morte em ambos os sexos no Brasil, e afetam quase a metade da população em algum momento durante a vida e levam à incapacidade 2 .

Segundo a Organização Mundial da Saúde (OMS) 13,5 milhóes de pessoas morreram de DCV em 2008, o que representa $30 \%$ de todas as mortes globais. Dessas mortes, 6,2 milhôes foram devido AVE $^{1}$. Estima-se que mais de $80 \%$ dos casos de eventos não fatais de AVE poderiam ter sido evitados ${ }^{3}$. O AVE é uma das maiores causas de mortalidade e inaptidão funcional, mas ainda não foi equacionado o impacto dessa doença com a implantação de políticas públicas eficazes destinadas a conter seu avanço ${ }^{4}$.

As açóes de maior impacto para o domínio da doença são as com finalidade de prevenção (primária e secundária) e direcionadas aos fatores de risco, chamados modificáveis. ${ }^{2,5}$ A prevalência do AVE é alta, e $90 \%$ dos sobreviventes desenvolvem algum tipo de deficiência ou incapacidade, com prejuízos nas funções sensitivas, motoras, de equilíbrio e de marcha, além do déficit cognitivo, disfágico e de linguagem, ${ }^{6} 15 \%$ dos pacientes não apresentam prejuízo da capacidade funcional, porém outros $85 \%$ necessitam de reabilitação e cuidados constantes, o que leva a um impacto econômico, social e familiar?.

Os pacientes que apresentam dificuldades físicas e/ ou neurológicas precisam de um processo dinâmico de reabilitaçáa, que deve ser realizado de maneira contínua e progressiva, e envolva a educação no intuito de atingir a restauração funcional, reintegração familiar e social, recuperando, assim, a qualidade de vida ${ }^{8}$.

As pessoas com alteraçóes funcionais secundárias ao AVE podem ser recebidas em unidades ambulatoriais, idealmente por equipe multiprofissional, composta por profissionais de várias áreas, como fisioterapeuta, fonoaudiólogo, médico, nutricionista, psicólogo, terapeuta ocupacional, assistente social, enfermeiro, farmacêutico, entre outros, que devem atuar de maneira interdisciplinar?.

As avaliações clínicas e instrumentais especializadas devem ser utilizadas na prática interdisciplinar, a fim de diagnosticar o nível de comprometimento, as potencialidades, e definir o plano de cuidados, fundamentado em métodos destinados à recuperação de déficits ou desenvolvimento de compensaçôes. A abordagem interdisciplinar contempla discussões periódicas sobre cada caso, incluindo as estratégias de atendimento. Dessa forma, a família receberá orientaçôes uniformes e de maneira consensual pela equipe, o que facilita $\mathrm{o}$ acesso e a adesão ao tratamento ${ }^{10}$. A reabilitação tem o intuito de capacitar os indivíduos e recuperaçâo dos déficits residuais do AVE, o que faz o indivíduo progredir ou manter o máximo grau de independência possível ${ }^{11}$.

Apesar de ter foco nos danos e prejuízos desencadeados pela patologia, é necessário também identificar e promover a reinserção social. Por essa razão, o trabalho em equipe representa uma possibilidade de favorecer o processo de reabilitação, e ampliar a atuação profissional na reabilitação global do indivíduo ${ }^{12}$.

Portanto, este estudo objetivou identificar na literatura evidências sobre a eficácia das intervençóes utilizadas por equipe interdisciplinar na reabilitação do paciente pós-acidente vascular encefálico, bem como os fatores que influenciam no acesso e entrega da reabilitação.

\section{Metodologia}

Foi realizada revisão integrativa da literatura, norteada pela questâo: Qual a efetividade das açôes realizadas pela equipe interdisciplinar na reabilitação do paciente pós-AVE, e quais fatores influenciam o acesso e entrega da reabilitação?

Para a realização da revisão integrativa adotaram-se os seguintes passos: seleção da questão temática, estabelecimentos de critérios de inclusão e exclusão dos artigos (seleção da amostra), definição dos dados a serem extraídos dos artigos selecionados, análise dos dados, interpretação dos resultados, e, por último, a apresentação da revisão. Esses passos foram empregados de acordo com o método de pesquisa desenvolvido por Broome e por Whittemore e Knafl ${ }^{13-14}$.

O levantamento de dados foi realizado no mês de dezembro de 2015, por meio de busca de artigos indexados nas bases de dados on-line Pubmed, Lilacs, Scielo e Cochrane Library. Com base em leituras prévias e no objetivo deste estudo, foram estabelecidos os seguintes descritores: Acidente vascular encefálico, reabilitação e equipe de assistência ao paciente.

Os critérios de inclusão para a seleção dos artigos foram: artigos publicados em inglês, português e espanhol, com resumos disponíveis na base de dados, com filtros para disponibilidade de textos gratuitos completos, com data de publicação de janeiro de 2010 a dezembro de 2015, em espécie humana. Foram excluídos 
os artigos que envolvessem equipe interdisciplinar e não tinham relação com a reabilitação do paciente pós-AVE.

A busca foi efetuada com três descritores em cada base de dados. Assim, ao empregar os descritores acidente vascular cerebral, reabilitação e equipe de assistência ao paciente obteve-se 1911 resultados no Pubmed, nenhum no Lilacs, 2 no Scielo em português, 2 no Cochrane Library.

De 1915 artigos encontrados, 12 foram selecionados - os demais não contemplavam os critérios de inclusão. Os artigos receberam numeração conforme sua ordem de publicação. Para a extração dos dados, utilizou-se um instrumento validado por Ursi ${ }^{15}$. Esse instrumento contempla os seguintes itens: identificação, instituição sede do estudo, tipo de revista científica, características metodológicas do estudo, avaliação do rigor metodológico e nível de evidência.

A análise dos dados ocorreu de forma descritiva e construiu-se uma tabela para apresentar a síntese dos artigos incluídos na revisão. Para a avaliação do nível de evidência do estudo, utilizou-se como referencial a classificação de Melnyk e Fineout-Overholt. Para o delineamento da pesquisa, utilizou-se os conceitos descritos por Polit, Beck e Hungler ${ }^{16-17}$.

\section{Resultados e discussão}

Os anos com maiores publicaçóes foram 2013 e 2014, com 9 artigos (62,9\%); a maioria publicada em periódicos internacionais. Os temas de atuação de uma equipe interdisciplinar na reabilitação do paciente pós-AVE foram publicados predominantemente em revistas da América do Norte, e apresentaram diferentes tipos de estudos, descritos nas tabelas 1 e 2. Para melhor compreensão dos resultados, os estudos foram classificados pelo tipo de abordagem. A Tabela 1 mostra artigos referentes à eficácia da reabilitação e a Tabela 2 mostra artigos referentes aos fatores que influenciam o acesso e realização dos serviços de reabilitação.

Tabela 1: Estudos referentes à eficácia da reabilitação segundo o ano, nível de evidência, tipos de estudo, objetivo e conclusão.

\begin{tabular}{|c|c|c|c|}
\hline Ano/Autor & Tipo de estudo & Objetivo & Conclusão \\
\hline $2012^{18}$ & $\begin{array}{l}\text { Revisão sistemática de } \\
\text { ensaios clínicos randomizados }\end{array}$ & $\begin{array}{l}\text { Investigar evidências da eficácia da } \\
\text { reabilitaçáo realizada por equipe } \\
\text { multidisciplinar em diferentes } \\
\text { problemas de saúde. }\end{array}$ & $\begin{array}{l}\text { Cuidados da equipe de reabilitação multidisciplinar } \\
\text { promoveram melhoras em populaçóes de idosos, idosos } \\
\text { com fratura de quadril, pessoas sem-abrigo com doença } \\
\text { mental, adultos com esclerose múltipla, AVE, lesão } \\
\text { cerebral, artropatia crônica, dor crônica, dor lombar e } \\
\text { fibromialgia. E necessária maior investigaçáo na área. }\end{array}$ \\
\hline $2013^{19}$ & Revisão sistemática & $\begin{array}{l}\text { Avaliar a eficácia do tratamento } \\
\text { multidisciplinar para pacientes com } \\
\text { AVE que vivem na comunidade. }\end{array}$ & $\begin{array}{l}\text { Há pouca evidência sobre a eficácia do atendimento } \\
\text { multidisciplinar no AVE na comunidade. Nenhum } \\
\text { estudo mostrou efeito na intervenção da vida diária, } \\
\text { dois relataram efeitos favoráveis na qualidade de vida. } \\
\text { Pesquisas adicionais, de maior qualidade metodológica, } \\
\text { devem fornecer mais detalhes no atendimento } \\
\text { multidisciplinar eficaz nessa populaçáo. }\end{array}$ \\
\hline $2013^{20}$ & Estudo longitudinal & $\begin{array}{l}\text { Determinar as limitaçóes e fatores } \\
\text { que influenciam o prognóstico de } \\
\text { pacientes com AVE em um centro de } \\
\text { reabilitaçáo. }\end{array}$ & $\begin{array}{l}\text { Houve melhora significativa na execução da tarefa } \\
\text { funcional de pacientes com AVE. Resultados mostram } \\
\text { alta prevalência de dependência no momento da alta. É } \\
\text { necessária execução da escala de avaliação funcional na } \\
\text { admissão, a fim de gerir as limitaçóes. }\end{array}$ \\
\hline $2014^{21}$ & $\begin{array}{l}\text { Estudo descritivo-analítico } \\
\text { e prospectivo-retrospectivo }\end{array}$ & $\begin{array}{l}\text { Avaliar a reabilitação, a prevalência } \\
\text { dos fatores de risco em AVE, } \\
\text { seus efeitos e propor medidas e } \\
\text { procedimentos para melhorar a } \\
\text { reabilitaçáo. }\end{array}$ & $\begin{array}{l}\text { Os fatores de risco mais comuns que afetam os } \\
\text { resultados da reabilitaçáo são a hipertensáo arterial } \\
(83 \%) \text { e diabetes }(33 \%) \text {. Deve-se combater esses } \\
\text { fatores de risco por meio da prevençáo primária e } \\
\text { secundária, e educaçáo do paciente sobre a detecçáo e } \\
\text { tratamento precoce desses fatores. }\end{array}$ \\
\hline $2014^{22}$ & Revisão & $\begin{array}{l}\text { Proporcionar aos profissionais de } \\
\text { terapia ocupacional evidências no } \\
\text { uso de intervençōes baseadas em } \\
\text { ocupação, e melhora da participaçáo } \\
\text { social pós-AVE }\end{array}$ & $\begin{array}{l}\text { A literatura evidencia o uso de intervenções baseadas } \\
\text { em ocupação para melhorar desempenho nas AVDs. } \\
\text { A evidência relacionada na área de ocupação deixa } \\
\text { lacunas que precisam ser abordadas em pesquisas } \\
\text { futuras, trabalhos nessa área precisam descrever } \\
\text { melhores intervençôes, controle adequado e utilizar } \\
\text { medidas para captar o efeito das intervençôes. }\end{array}$ \\
\hline $2014^{23}$ & $\begin{array}{l}\text { Estudo prospectivo } \\
\text { único centro de coorte }\end{array}$ & $\begin{array}{l}\text { Avaliar a viabilidade e eficácia da } \\
\text { reabilitação neurológica e prevenção } \\
\text { no ataque isquêmico transitório } \\
\text { (AIT) ou AVE com ou sem déficits } \\
\text { residuais. }\end{array}$ & $\begin{array}{l}\text { Houve melhora significativa da capacidade de } \\
\text { exercício, tabagismo, pressáo sistólica e diastólica, } \\
\text { índice de massa corporal, lipoproteína de baixa } \\
\text { densidade, colesterol, triglicerídeos e qualidade de vida. } \\
\text { Dessa forma, a reabilitação ambulatorial cardiovascular } \\
\text { e neurológica abrangente é viável e eficaz. }\end{array}$ \\
\hline
\end{tabular}


Tabela 2: Estudos referentes aos fatores que influenciam o acesso e realização da reabilitação segundo o ano, nível de evidência, tipos de estudo, objetivo e conclusão.

\begin{tabular}{|c|c|c|c|}
\hline Ano/Autor & Tipo de estudo & Objetivo & Conclusão \\
\hline $2012^{24}$ & $\begin{array}{l}\text { Coorte prospectivo } \\
\text { observacional }\end{array}$ & $\begin{array}{l}\text { Identificar fatores na tomada de } \\
\text { decisão sobre a idoneidade para } \\
\text { a reabilitação pós-AVE grave na } \\
\text { fase aguda. }\end{array}$ & $\begin{array}{l}\text { Importante para aceitar o paciente na reabilitaçáo: } \\
\text { cogniçáo, mobilidade e comunicaçáo pré-mórbidas. } \\
\text { Os resultados poderão ser utilizados num modelo } \\
\text { para melhorar a equidade de acesso à reabilitaçáo } \\
\text { após o AVE grave. }\end{array}$ \\
\hline $2013^{25}$ & Estudo transversal & $\begin{array}{l}\text { Examinar a implantação, } \\
\text { aceitabilidade e benefícios no } \\
\text { estabelecimento de metas e } \\
\text { planejamento de açóes (GAP) } \\
\text { de equipe de reabilitação na } \\
\text { comunidade com pessoas em } \\
\text { recuperaçáo de AVE. }\end{array}$ & $\begin{array}{l}\text { O GAP foi implantado conforme protocolo, fases de } \\
\text { avaliaçáo e feedback. Duas questôes foram levantadas } \\
\text { em relação ao GAPs: Os profissionais se preocupam } \\
\text { com o impacto da meta não-alcançada, bem-estar e } \\
\text { registro adequado do paciente? O GAP foi considerado } \\
\text { benéfico e aceitável na equipe de reabilitaçáo? }\end{array}$ \\
\hline $2013^{26}$ & Estudo Coorte & $\begin{array}{l}\text { Investigar as características } \\
\text { sociodemográficas e clínicas dos } \\
\text { pacientes com AVE, quem recebe } \\
\text { o tratamento e reabilitação quando } \\
\text { saem do cuidado agudo neurológico. }\end{array}$ & $\begin{array}{l}40,8 \% \text { dos pacientes com AVE foram submetidos à } \\
\text { reabilitação neurológica ou geriátrica. Aqueles com } \\
\text { paresia e disartria apresentam maiores chances de } \\
\text { inclusão na reabilitaçáo; pacientes com AVE anterior } \\
\text { são menos propensos a reabilitação. A exclusão em } \\
\text { idade avançada deve ser bem avaliada. }\end{array}$ \\
\hline $2014^{27}$ & Estudo qualitativo & $\begin{array}{l}\text { Explorar a percepção de reabilitação } \\
\text { em longo prazo entre os profissionais } \\
\text { de reabilitaçáo e de pessoas com } \\
\text { AVE, e identificar estratégias para a } \\
\text { prestaçáo de tais serviços. }\end{array}$ & $\begin{array}{l}\text { Obstáculos à prestação de serviços de reabilitação AVE } \\
\text { em longo prazo são multifatoriais. Criaçáo de centros } \\
\text { de reabilitação de base comunitária e treinamento } \\
\text { para a família no domicílio, são estratégias potenciais } \\
\text { para permitir a continuação da reabilitação do AVE } \\
\text { de longa duraçáo. }\end{array}$ \\
\hline $2014^{28}$ & Estudo transversal & $\begin{array}{l}\text { Explorar em que medida as equipes } \\
\text { médicas estâo atendendo os níveis } \\
\text { de pessoal recomendados. }\end{array}$ & $\begin{array}{l}\text { Apenas } 42 \% \text { das equipes alcançaram a diretriz do } \\
\text { Departament of Health (DoH) para fisioterapia, } \\
\text { menos de } 16 \% \text { chegaram à diretriz para fala e } \\
\text { terapia de linguagem, e } 84 \% \text { orientação para terapia } \\
\text { ocupacional. A maioria das unidades de AVE está } \\
\text { operando abaixo das diretrizes DoH e são desafiadas } \\
\text { a fornecer a quantidade de terapia, tempo de } \\
\text { atendimento para a recuperaçấo funcional ideal para } \\
\text { pacientes com AVE. }\end{array}$ \\
\hline $2015^{29}$ & $\begin{array}{l}\text { Estudo multicêntrico, } \\
\text { exploratório qualitativo. }\end{array}$ & $\begin{array}{l}\text { Relatar as opiniōes da equipe } \\
\text { em realizar uma intervenção } \\
\text { de reabilitaçáo complexa: o } \\
\text { que levou o paciente a terapia } \\
\text { durante a internação por AVE. }\end{array}$ & $\begin{array}{l}\text { Surgiram cinco assuntos: efeitos das intervençóes, } \\
\text { problemas práticos, fatores relacionados aos pacientes } \\
\text { e aos profissionais e competência para desenvolver } \\
\text { habilidades. Para atingir os protocolos de atendimento } \\
\text { é necessário tempo, habilidades, confiança, estratégias } \\
\text { de tratamento e adaptaçáo das necessidades de cada } \\
\text { indivíduo. }\end{array}$ \\
\hline
\end{tabular}

Portanto, serviços são desafiados a fornecer a quantidade recomendada de terapia e o tempo de atendimento para facilitar a recuperação funcional, além de estarem bem organizados para fornecer reabilitação aguda e intermediária.

\section{Discussão}

De modo geral, a reabilitaçáo no AVE compreende três etapas, que perpassam prevenção, tratamento agudo e reabilitação. ${ }^{23} \mathrm{O}$ principal objetivo da reabilitação é maximizar a independência funcional, facilitar a reintegração a aumentar a participação em rotinas de vida ${ }^{30}$.

Quanto ao enfoque temático, pode-se observar em todos os artigos selecionados que as açóes necessárias na reabilitaçấo do paciente com AVE devem levar em consideração fatores sociais, condições pré-morbidas além de funçóes pós-AVE, indispensáveis na tomada de decisão para realizar a reabilitação ${ }^{24}$. É importante considerar ações direcionadas nos fatores de risco para HAS e DM por meio da prevenção primária e secundária, e educação do paciente sobre a detecção e tratamento precoce desses fatores de risco ${ }^{21}$.

Para alcançar uma reabilitação que atenda a todas essas demandas pessoais, é necessário tratamento sistemático, prestado por equipe interdisciplinar bem estruturada, para, assim, atingir melhores resultados funcionais, com base em estudos científicos atuais, por isso a importância dos estudos clínicos para melhorar o conhecimento das estratégias e alcançar a eficácia da reabilitação ${ }^{31}$.

São múltiplos os fatores que culminam em obstáculos à prestação de serviços de reabilitação pós-AVE em 
longo prazo, mas uma solução viável e que permita a continuação da reabilitação de longa duração poderia ser a criação de centros de reabilitação que incluam a participação da família, estimulem e conduzam o tratamento em nível domiciliar. ${ }^{32}$

Apesar dessas estratégias, permanece inconclusiva a pergunta sobre o que seria o momento ideal de "reabilitaçấo de longo prazo" para sobreviventes de AVE. Questiona-se que reabilitação de longa duração significaria maior custo aos serviços de saúde. Porém, estudos recentes têm mostrado que, devido à plasticidade do cérebro, a recuperação funcional é possível durante muitos anos após o AVE, embora a recuperação neurológica seja atingida no seu nível máximo no prazo de três meses $^{33}$. Orientações de reabilitação com embasamento teórico recomendam que sobreviventes de AVE devem ter acesso à reabilitação enquanto benefícios ou melhora forem perceptíveis ${ }^{22-32}$. Pesquisas futuras devem investigar a necessidade de orientar a tomada de decisôes relacionadas com serviço de reabilitaçáo em longo prazo para as pessoas que tiveram AVE.

Estudos relacionados às açóes da equipe multidisciplinar na reabilitação após AVE mostram que, apesar da heterogeneidade dos cuidados, a atuação da equipe multidisciplinar pode melhorar efetivamente a intervenção de reabilitaçáa ${ }^{33}$. As dificuldades para a equipe multidisciplinar permeiam entre a falta de profissionais, falta de consciência entre pacientes e cuidadores, educação insuficiente e falta de serviços especializados para reabilitação de pacientes com $\mathrm{AVE}^{31}$. As dificuldades enfrentadas pelos profissionais podem ser explicadas pela pouca evidência científica da eficácia do atendimento multidisciplinar para pacientes com AVE, em especial em domicílio ${ }^{19}$.

Estudos que demonstram intervençôes eficazes destacam a importância de modelos futuros que levem em consideração a função pré-mórbida e fatores sociais para a seleção de pacientes em reabilitação pós-AVE. Os dados indicam que a maioria das unidades que atendem pacientes pós-AVE opera abaixo das diretrizes priorizadas pelo Ministério da Saúde, o que os desafia a avaliar a quantidade e qualidade de terapia oferecida ao paciente, e assim chegar em um nível considerado ideal ${ }^{28}$.

$\mathrm{O}$ resultado dessa dificuldade em formar equipes multidisciplinares, bem como divulgar estudos acerca da eficácia das intervençóes, gera uma problemática ainda maior. Considerando a organizaçáo dos serviços de saúde de maneira geral, ainda existem barreiras que dificultam o acesso diante das necessidades apresentadas pelos indivíduos. Embora o modelo de organização preconize um fluxo articulado e organizado, observam-se fragilidades nos caminhos de acesso aos serviços especializados, bem como indisponibilidade de serviços de reabilitação ${ }^{34}$. De fato, atualmente são poucos os serviços que oferecem rotina de acompanhamento após seis meses de AVE, e isso pode ter contribuído para evidenciar lacunas em relação à pesquisa sobre o tema.

Essa revisão demonstra que ainda há necessidade de estudos de qualidade metodológica relativos à avaliação da eficácia dos diferentes tipos de atendimento multidisciplinar para reabilitar pacientes com AVE após a alta hospitalar, pois a maioria dos estudos foi baseada em programas específicos e pesquisas sobre os efeitos da reabilitação apenas em determinadas funções (físicas, cognitivas ou fala) e não nos efeitos da assistência multidisciplinar para os pacientes com AVE.

\section{Conclusão}

Estudos indicam que a reabilitação iniciada no primeiro trimestre pós-AVE torna-se mais eficiente na recuperação da funcionalidade física, comunicativa e cognitiva do que a reabilitação tardia. Os fatores decisivos para incluir o indivíduo na reabilitação constituem-se da condição pré-mórbida e função pós-AVE; para exclusão, foram idade avançada e história prévia de AVE. Entretanto, mais estudos com rigor científico precisam ser realizados para comprovar outros fatores que possam interferir o acesso do indivíduo à reabilitação e a eficácia da reabilitação pós-AVE realizada pela equipe multidisciplinar.

Além da pesquisa sobre a continuidade dos cuidados de reabilitação, é necessário descobrir intervenções que melhorem a reintegração do paciente à comunidade, e divulgar amplamente os resultados dos estudos, a fim de melhorar a visibilidade e reconhecimento da base científica de práticas clínicas. Portanto, diante das lacunas evidenciadas e os resultados apontados nos artigos incluídos nesta revisão integrativa, entende-se ser necessário intensificar esforços para o desenvolvimento de novas pesquisas com delineamentos que produzam evidências fortes relativas ao tema investigado, principalmente na realidade da prática da reabilitação pós-AVE.

\section{Referências}

1. World Health Organization. Cardiovascular diseases (CVDs) [Internet]. Geneva: WHO; 2013 [citado em 2016 jan 22]. Disponível em: https://goo.gl/A2sC

2. Jorge MHPM, Laurenti R, Lima-Costa MF, Gotlieb SLD. A mortalidade de idosos no Brasil: a questão das causas mal definidas. Epidemiol serv. saúde [Internet]. 2008 [citado em 2016 jan 22];17(4):271-81. Disponível em: https:/goo.gl/ XejxKv

3. Goldstein LB, Bushnell CD, Adams RJ, Appel LJ, Braun LT, Chaturvedi $S$, et al. Guidelines for the primary 
prevention of stroke: a guideline for healthcare professionals from the American Heart Association/American Stroke Association. Stroke [Internet]. 2011. [citado em 2016 jan 12];42(2):517-84. Disponível em: https://goo.gl/q6MUUe

4. Pontes-Neto OM, Silva GS, Feitosa MR, de Figueiredo NL, Fiorot JA Jr, Rocha TN, et al. Stroke awareness in Brazil: Alarming results in a community-based study. Stroke [Internet]. 2008 [citado em 2016 jan 12];39(2):292-6. Disponível em: https://goo.gl/x99kFd

5. Kernan WN, Ovbiagele B, Black HR, Bravata DM, Chimowitz MI, Ezekowitz MD, et al. Guidelines for the prevention of stroke in patients with stroke or transient ischemic attack: A guideline for healthcare professionals from the American Heart Association/American Stroke Association. Stroke [Internet]. 2011 [citado em 2016 jan 12];42(1):227-76. Disponível em: https://goo.gl/0E7IlA

6. Ryerson S, Byl NN, Brown DA, Wong RA, Hidler JM. Altered trunk position sense and its relation to balance functions in people post-stroke. J neuro phys ther [Internet]. 2008. [citado em 2016 jan 12];32(1):14-20. Disponível em: https://goo.gl/44vXau

7. Cruz KCT, D'Elboux DMJ. Evaluation of functional capaciy in elders with encephalic vascular accident. Acta paul enferm [Internet]. 2009 [citado em 2016 feb 4];22(5):66672. Disponível em: https://goo.gl/scJ6hl

8. Scalzo PL, De Souza ES, Moreira AGO, Vieira DAF. Qualidade de vida em pacientes com Acidente Vascular Cerebral: clínica de fisioterapia. Rev. neurociênc. (Impr.) [Internet]. 2008 [citado em 2016 jan 22];18(2):139-44. Disponível em: https://goo.gl/IkhJIS

9. Brasil. Ministério da Saúde. Secretaria de Atenção à Saúde. Departamento de Açôes Programáticas Estratégicas. Diretrizes de atenção à reabilitaçáo da pessoa com acidente vascular cerebral. Brasília, DF: Ministério da Saúde; 2013.

10. Brasil. Ministério da Saúde. Secretária de Atenção à Saúde. Diretrizes de Atenção à Reabilitação da Pessoa com Acidente Vascular Cerebral. Brasília, DF: Ministério da Saúde; 2012.

11. European Stroke Organisation Executive Committee, European Stroke Organisation Writing Committee. Guidelines for management of ischaemic stroke and transient ischaemic attack 2008. Cerebrovasc Dis. 2008; 25(5):457-507.

12. Queiroz E, Araujo TCCF. Trabalho de equipe em reabilitação: um estudo sobre a percepção individual e grupal dos profissionais de saúde. Paidéia (Ribeirão Preto) [Internet]. 2009 [citado em 2016 jan 12];19(43):177-87. Disponível em: https://goo.gl/tUHCwf

13. Broome ME. Integrative literature reviews for the development of concepts. Concept development in nursing: foundations, techniques and applications. Philadelphia (USA): WB Saunders Company; 2000. p. 231-50.

14. Whittemore R, Knafl K. The integrative review: updated methodology. Arch phys med rehabil [Internet]. 2005 [citado em 2016 jan 22];52(5):543-56. Disponível em: https:// goo.gl/zyAEEo
15. Ursi ES, Gavão CM. Prevenção de Lesôes de Pele no Perioperatório: revisão integrativa de literatura. Rev latinoam enferm [Internet]. 2006 [citado em 2016 jan 22];14(1):124-31. Disponível em: https://goo.gl/k5MEvM

16. Melnyk BM, Fineout-Overholt E. Making the case for evidence-based practice. In: B. M. Melnyk, \& FineoutOverholt, Evidence-based practice in nursing $\&$ healthcare. A guide to best practice. Philadelphia: Lippincot Williams \& Wilkins; 2005.

17. Polit DF, Beck CT, Hungler BF. Fundamentos de pesquisa e enfermagem, métodos, avaliação e utilização. 5 ed. Porto Alegre: Armed. 2004.

18. Momsen AM, Rasmussen JO, Nielsen CV, Iversen MD, Lund H. Multidisciplinary team care in rehabilitation: an overview of reviews. J rehabil med [Internet]. 2012 [citado em 2016 jan 04];44(11):901-12. Disponível em: https://goo.gl/1Ex9mt

19. Fens M, Vluggen T, van Haastregt JC, Verbunt JA, Beusmans $\mathrm{GH}$, van Heugten CM. Multidisciplinary care for stroke patients living in the community: a systematic review. J rehabil med [Internet]. 2013 [citado em 2016 jan 04];45(4):321-30. Disponível em: https://goo.gl/v1RYAs

20. Joseph C, Rhoda A. Activity limitations and factors influencing functional outcome of patients with stroke following rehabilitation at a specialised facility in the Western Cape. Afr Health Sci [Internet]. 2013 [citado em 2016 jan 02];13(3):646-54. Disponível em: https://goo.gl/IiLcUk

21. Tanovic E, Selimovic S, Tanovic H. Assessment of the effects of rehabilitation after cerebrovascular accident in patients with diabetes mellitus and hypertension as risk factors. Med Arch [Internet]. 2014 [citado em 2016 jan 02];68(2):12427. Disponível em: https://goo.gl/RTprV2

22. Wolf TJ, Chuh A, Floyd T, McInnis K, Williams E. Effectiveness of occupation-based interventions to improve areas of occupation and social participation after stroke: an evidence-based review. Am J Occup Ther [Internet]. 2015 [citado em 2016 Jan 02];69(1):6901180060p1-11. Disponível em: https://goo.gl/ebOi4t

23. Lindsay P, Bayley M, Hellings C, Hill M, Woodbury E, Phillips S. Canadian best practice recommendations for stroke care. CMAJ [Internet]. 2008 [citado em 2016 jan 2];179(12 Suppl):S1-S25. Disponível em: https:/goo.gl/ JHq6nw

24. Hakkennes SJ, K Brock, Hill KD. Selection for inpatient rehabilitation after acute stroke: a systematic review of the literature. Arch phys med rehabil [Internet]. 2011 [citado em 2016 jan 22];92(12):2057-70. Disponível em: https:// goo.gl/40dCLE

25. Scobbie L, McLean D, Dixon D, Duncan E, Wyke S. Implementing a framework for goal setting in community based stroke rehabilitation: a process evaluation. BMC Health Serv Res [Internet]. 2013 [citado em 2016 jan 22];13(1):190. Disponível em: https://goo.gl/GrRYsT

26. Unrath $M$, Kalic M, Berger $K$. Who receives rehabilitation after stroke?: Data from the quality assurance project 
“Stroke Register Northwest Germany”. Dtsch Arztebl Int [Internet]. 2013 [citado em 2016 jan 22];110(7):101-7. Disponível em: https://goo.gl/gGUIma

27. Mohd Nordin NA1, Aziz NA, Abdul Aziz AF, Ajit Singh DK, Omar Othman NA, Sulong S, Aljunid SM. Exploring views on long term rehabilitation for people with stroke in a developing country: findings from focus group discussions. BMC Health Serv Res [Internet]. 2014 [citado em 2016 jan 24];14(1):118. Disponível em: https://goo.gl/fLzZKY

28. McHugh G, Swain ID. A comparison between reported therapy staffing levels and the department of health therapy staffing guidelines for stroke rehabilitation: a national survey. BMC Health Serv Res. [Internet]. 2014 [citado em 2016 jan 24];14(1):216. Disponível em: https://goo.gl/YTX9rK

29. Horne M, Thomas N, Vail A, Selles R, McCabe C, Tyson S. Staff's views on delivering patient-led therapy during inpatient stroke rehabilitation: a focus group study with lessons for trial fidelity. Trials [Internet]. 2015 [citado em 2016 jan 24];16(1):137. Disponível em: https://goo.gl/Egj07C
30. National Stroke Foundation (AUS). Clinical Guidelines for Stroke Management. Melbourne, Australia; National Stroke Fundation; 2010.

31. Knecht S, Hesse S, Oster P. Rehabilitation after stroke. Dtsch arztebl int [Internet]. 2011 [citado em $2016 \mathrm{feb}$ 04];108(36):600-6. Disponível em: https://goo.gl/jak32a

32. Kannel WB. Blood pressure as a cardiovascular risk factor: prevention and treatment. JAMA. [Internet]. 1996 [citado em 2016 jan 22];275(20):1571-6. Disponível em: https:// goo.gl/m8XXjP

33. Momsen AM, Rasmussen JO, Nielsen CV, Iversen MD, Lund H. Multidisciplinary team care in rehabilitation: an overview of reviews. J Rehabil Med [Internet] 2012 [citado em 2016 jan 22];44(11):901-12. Disponível em: https:// goo.gl/9f1T3g

34. Gadelha IDS, Brito GE, Nascimento JA do et al. Tempo de acesso aos serviços de reabilitação de pessoas acometidas por ave. Rev enferm UFPE [Internet] 2015 [citado em 2016 jan 22];9(12):1246-24. Disponível em: https://goo.gl/xznAIB

Como citar este artigo:

Vieira DCD, Callegaro CC, Bittencourt VLL, Silva TP, Winkelmann ER. Reabilitação de acidente vascular encefálico: revisão de literatura. Rev. Aten. Saúde. 2017;15(52):89-95. 